\title{
CORRIGENDUM AND ADDENDUM
}

\section{to the paper \\ AN INFINITE CONSTRUCTION IN RING THEORY}

\author{
by E. A. WHELAN
}

(Received 15 September, 1989; revised 26 March, 1990)

1. Point (3) of the main theorem of our paper [3, Theorem 1.1] is incorrect: this note corrects the main and consequential errors, and shows that (after minor adjustments) almost all the other results of [3], including the remaining seven points of Theorem 1.1, remain correct.

2. The theme of [3] was a family of functors $G_{t}(-)$, defined on the category of rings with unity for each cardinal $t$. For $t=0,1$, the results of [3] are unchanged, but, for $2 \leqslant t<\infty$, major, and, for $t$ infinite, less major, corrections are necessary; we therefore assume $2 \leqslant t$. Terminology and notation are standard or as in [3], and I would like to thank A. W. Chatters and an anonymous referee for comments which prompted this correction.

3. In [3, p. 350] we defined the (functorial) ring extensions $R \rightarrow G_{t}(R)$ using an index set $B$ such that $\operatorname{card}(B)=b \geqslant t$ is an infinite cardinal, and a family $T_{1}$ of injective mappings $\sigma: B \rightarrow B$ such that:

(a) $\forall \sigma \in T_{1}, \operatorname{card}(B \backslash \operatorname{Im}(\sigma))=b$;

(b) $\forall \sigma, \tau \in T_{1},(\operatorname{Im}(\sigma) \cap \operatorname{Im}(\tau) \neq \varnothing) \Rightarrow \sigma=\tau$. To these axioms we must now add the stipulation (incorrectly treated as optional in [3]) that:

(c) if $t \geqslant 2, B=\bigcup_{\sigma \in T_{1}} \operatorname{Im}(\sigma)$, i.e. $\left\{\operatorname{Im}(\sigma): \sigma \in T_{1}\right\}$ is a complete set of equivalence classes (each of cardinality $b$ ) in $B$.

4. As in [3], let $H_{1}$ be the unital monoid of injective mappings $B \rightarrow B$ generated by $T_{1}, H_{2}=\left\{\operatorname{Im}(\tau): \tau \in H_{1}\right\}$. Additionally, for $n \geqslant 0$, let $X_{n}$ be the subset of mappings comprising the products of exactly $n$ elements of $T_{1}$ and $Y_{n}=\left\{\operatorname{Im}(\tau): \tau \in X_{n}\right\}$ for $n \geqslant 0$. Suppose that $M$ is as in [3], that, for each $i \in B$ and each pair $I, J \in H_{2}, x_{i} \in M$, $E_{I J} \in \operatorname{End}\left(M_{R}\right)$ are also as in [3], and that the ring $G=G_{t}(R)$ is (still) the subring of End $\left(M_{R}\right)$ generated by $\mathbb{E}=\left\{E_{I J}: I, J \in H_{2}\right\}$ plus the left multiplications by the elements of $R$.

5. From [3],

(a) each $E_{I J}$ centralizes $R$;

(b) $\mathbb{E} \cup\{0\}$ is multiplicatively closed;

(c) $E_{B B}=1_{G}=\mathrm{id}_{M}$;

(d) for all $I, J \in \Omega, E_{B B}=E_{B I} E_{I J} E_{J B}$;

(e) for all $I, J, K, L \in \Omega, E_{I J} E_{K L}=0$ if and only if $J \cap K=\varnothing$;

(f) if $t \geqslant 2$ then for every $n \geqslant 1$ and every $I \in X_{n}$ there exists $J \in X_{n}$ such that $I \cap J=\varnothing$. 
6. The error in Theorem 1.1(3) of [3] is the claim that $G=G_{t}(R)$ is left and right free on $\mathbb{E}$. To get a counter-example, take $t=2, T_{1}=\{\sigma, \tau\}$, and $I=\operatorname{Im}(\sigma), J=\operatorname{Im}(\tau)$, so that $B=I \cup J$ and $I \cap J=\varnothing$. Then $E_{l l}, E_{J J}$ are orthogonal idempotents and $E_{I I}+E_{J J}=E_{B B}=\mathrm{id}_{M}$.

7. The purported proof of 'freeness' is at [3,2.3], but depends crucially on $[3,2.2(d)]$, which claims without specific proof that a form of partial cancellation holds in $\mathbb{E}$. The following is an easy counter-example: for any $t \neq 0$, if $J \in \Omega, J \neq B$, then $E_{B J} E_{J J}=E_{B J} E_{B B}=E_{B J} \neq 0$.

8. For infinite $t$, the new assumption (c) of Section 3 makes no practical difference, but for $2 \leqslant t<\infty$, it ensures that the idempotent $1-\sum_{I \in Y_{n}} E_{I I}$ is zero for every $n \geqslant 0$.

9. Whether $t$ is finite or infinite, it is (now) easy to check that, for each $n \geqslant 0$, $E_{I J} E_{K L}=E_{I L} \delta_{J K}$ for all $I, J, K, L \in Y_{n}$, i.e. each set $\mathbb{E}_{n}=\left\{E_{I J}: I, J \in Y_{n}\right\}$ is a set of matrix units over $R$ of degree $t^{n}$. Thus, using the condition mentioned in Section 8 , we have the following result.

Proposition 1. If $2 \leqslant t<\infty$ and $n \geqslant 0$ :

(a) the bimodule $G(n)=R \mathbb{E}_{n}=\mathbb{E}_{n} R$ is a subring of $G_{t}(R)$, isomophic (over $R$ ) to $M_{t^{n}}(R)$;

(b) $\mathbb{E}_{n} \subseteq \mathbb{Z}_{\mathbb{E}_{n+1}}$;

(c) hence $R=G(0) \subset G(1) \quad\left(\simeq M_{t}(R)\right) \subset \ldots \subset G(n) \quad\left(\simeq M_{t^{n}}(R)\right) \subset \ldots$ is a strictly ascending chain of subrings of $G=G_{t}(R)$, with union $G$.

10. It now follows easily that, for $t<\infty$, the other points of Theorem 1.1 of [3] are correct, as are the results of Section 4 and of Section 3 excluding 3(iv). The problems concern one-sided ideals, where our "proofs" made extensive but implicit use of freeness. Using the subrings $G(n)$ it follows that, if $A \subset B$ are right ideals of $R$ then $A G \subset B G$, and hence (correcting Sections 3(iv) and 5 of [3]) we have the following theorem.

THEOREM 2. (a) If $R$ is right primitive then so is $G=G_{t}(R)$;

(b) $J(R)=R \cap J(G)$ and $J(G)=G J(R)=J(R) G$.

We do not know if the converse to Theorem 2(a) is true. Clearly, however, every prime of $R$ is an intersection of maximal, right primitive or quasi-primitive ideals (see [4] for the latter definition) if and only if the same holds in $G_{t}(R)$.

11. Using the rings $G(n)$ it is also possible for us to obtain information about the structure and isomorphism classes of the ring extensions $R \rightarrow G_{t}(R), 2 \leqslant t<\infty$. If $n \in \mathbb{N}$, let $\mathrm{V} n$ denote the product of the distinct prime divisors of $n$.

Proposition 3. If $2 \leqslant t \leqslant \infty$ then $G_{t}(R)$ and $G_{\sqrt{t}}(R)$ are isomorphic as $R$-algebras to the tensor product (over $R$ )

$$
H=G_{p(1)}(R) \otimes G_{p(2)}(R) \otimes \ldots \otimes G_{p(r)}(R),
$$

where $p^{(1)}, p^{(2)}, \ldots, p^{(r)}$ are the distinct prime divisors of $t$. 
We observe that each extension $R \rightarrow G_{p(i)}(R)$ has no non-trivial tensor product decomposition (over $R$ ), that the decomposition as a product of such indecomposables in Proposition 3 is unique, and that ring extensions resembling these have been discussed at various places in the literature, e.g. [1, p. 341]. Finally, we note that (contrary to $\left[3\right.$, p. 351]) if $2 \leqslant t(1), t(2)<\infty$ then $G_{t(1)}(R)$ embeds over $R$ in $G_{t(2)}(R)$ if and only if $\sqrt{t}(1)$ divides $\sqrt{t}(2)$.

12. Apart from the error over cancellation (see Section 7 above), most of the rest of [3] remains correct in the case that $t$ is infinite. In particular, "freeness" (Theorem 1.1(3) of [3]) is correct, and a proof may be found in Theorem 2.3 of [2]. For infinite $t$, the only further errors in [3] concern embeddings (where $G_{t(1)}(R)$ does not embed over $R$ in $G_{t(2)}(R)$ when $2 \leqslant t(1)<t(2)$ and $t(2)$ is infinite), and the discussion of the Jacobson radical in [3,5.1, 5.2 and 5.3]. This discussion becomes correct if the assertions just cited are amended to stipulate that $t$ is infinite (though a drafting error must also be eliminated: in $[3,5.1]$ the term "right quasi-inverse" should read "right inverse".

13. By ([3, Theorem $1.1(8)]$, if $2 \leqslant t$ each ring $G=G_{t}(R)$ has the pleasing property that every finitely generated one-sided $G$-module is cyclic. It is not difficult to establish the analogous property for bimodules: if $t(1), t(2)>1$ are cardinals, $S, R$ are rings and $G=G_{t(1)}(S), H=G_{t(2)}(R)$ then every finitely generated $H-G$ bimodule is principal (as a bimodule).

\section{REFERENCES}

1. M. K. Smith, Group actions on rings: some classical problems, in F. van Oystaeyen (ed.), Methods in ring theory (D. Reidel, 1984), 327-346.

2. E. A. Whelan, Another infinite construction in ring theory, submitted to Quart. J. Math. Oxford.

3. E. A. Whelan, An infinite construction in ring theory, Glasgow Math. J. 30 (1988), 349-357.

4. E. A. Whelan, The symmetric ring of quotients of a primitive ring is primitive, Comm. Algebra 18 (1990), 615-633.

School of Mathematics

UNIVERSITY OF EAST ANGLIA

NORWICH, NORFOLK NR4 7TJ

ENGLAND 